12 DIMENSIONS OF BIDIRECTIONAL TRUST: RELATIONSHIPS IN NURSES' CARING FOR ADOLESCENTS AND EMERGING ADULTS

J Evered. University of Wisconsin-Madison, USA

\subsection{6/bmjopen-2021-QHRN.12}

Background Many social actors participate in the health and social care of adolescents and emerging adults with life-limiting illnesses or injuries. Though nurses bear intimate witness to the experiences of young people and their loved ones, dimensions of social relationships between nurses, young people, and members of young peoples' social circles lack description and interpretation in extant literature.

Aim The purpose of this inquiry was to develop a situationspecific theory to explain the nature of social relationships in nurses' caring for adolescents and emerging adults with lifelimiting illnesses or injuries. This paper focuses on dimensions of trust, offering insight into how bidirectional trust between a nurse and the social actors of a young person's circle determines the nature of nurses' caring.

Method In alignment with dimensional analysis method, theoretical sampling, data collection, and data analysis occurred iteratively and concurrently. Twenty-one nurses who provide care in pediatric or adult inpatient, outpatient, and home care settings in the United States participated in in-depth semistructured interviews. Inductive coding, dimensionalization, and constant comparison technique helped surface and relate dimensions of social relationships in nurses' caring for adolescents and emerging adults.

Findings Trust is both a dimension of relational closeness and a driver of nurses' care for adolescents and emerging adults. Bidirectional trust evolves as nurses assess, diagnose, and reevaluate the young person, the social circle, and themselves. Nurses' trust in other social actors determines how nurses conceptualize and perform relational care.

Conclusions When and how nurses involve social actors in the health and social care of a young person depends on nurses' feelings of trust. Understanding the dynamic bidirectional nature of trust holds implications for nurses' enactment of relationship-centered care in the context of young peoples' life-limiting illnesses and injuries.

\section{DOES THE 'CANADA COVID-19 ALERT' APP STAND UP TO CRITICAL SCRUTINY? A RAPID QUALITATIVE ASSESSMENT}

\begin{abstract}
1,2,30 Gómez-Ramírez, 3,4p Medeiros, 5,6R Wainer, ${ }^{2}$ I lyamu. 'British Columbia Centre for Disease Control, Vancouver BC, Canada; ${ }^{2}$ School of Population and Public Health, University of British Columbia, Vancouver BC, Canada; ${ }^{3}$ CIHR Canadian HIV Trials Network, Vancouver BC, Canada; ${ }^{4}$ Women's College Hospital, Toronto ON, Canada; ${ }^{5}$ Department of Sociology, University of British Columbia, Vancouver BC, Canada; ${ }^{6}$ Department of Anthropology, University of British Columbia, Vancouver BC, Canada
\end{abstract}

\subsection{6/bmjopen-2021-QHRN.13}

Background In the wake of the urgency to respond to the COVID-19 pandemic, several tools have been rapidly developed and implemented. As part of the 'digital government response to COVID-19,' the Government of Canada launched the 'Canada COVID-19 Alert' app in June 2020. This cellphone/tablet-based app aims to use Bluetooth technology to notify users of potential exposures to COVID-19. Despite reports that over 40 such apps have been implemented worldwide, evaluations of the government's intended aims, the extent to which those aims are realized through these tools, and the implications of their usage are missing.

Objective This study undertakes a rapid qualitative assessment of the Canada COVID-19 app during the first phase of its development and implementation (May-October 2020).

Methods Three qualitative methods are employed: 1) Review of government website and available documentary materials to assess intended aims. 2) Walkthrough of the app to assess the extend to which the intended aims might be accomplished. 3) Analysis of Canadian newspaper coverage to assess the societal implications of app usage. Critical analyses are guided by asking: for what ends, for whom, by whom, and under which circumstances can the app operate as intended. Rapid assessment focuses on the role and balance of evidence-, needs-, and/or technology-driven justifications, and of public healthand/or government-oriented goals and functions.

Results Preliminary analyses indicate that the app's aims remain elusive. It serves a limited potential proximity notification role, rather than a contact tracing aim. Debates about data privacy and storage concerns indicate scepticism and mistrust of the app. Profit (re)distribution and tool de-implementation plans amid an unlikely clear-cut pandemic 'end' remain absent.

Conclusions In a time which trust in public health measures are essential for the control of communicable disease, the tools employed must be assessed. Only through critical scrutiny can new tools stand up to benefit the public good.

\section{THE DYNAMICS OF CO-DESIGNING A HYBRID REALIST- PARTICIPATORY EVALUATION WITHIN YOUTH PEER SUPPORT SERVICES}

${ }^{1} \mathrm{~T}$ Halsall, ${ }^{2} \mathrm{M}$ Daley. 'University of Ottawa Institute of Mental Health Research, Canada; ${ }^{2}$ LOFT Community Services, Canada

\subsection{6/bmjopen-2021-QHRN.14}

Peer support services are grounded in the approach of involving 'peers' or individuals who share key lived experiences with clients. Although there is an expanding body of literature focused on youth peer support, there continues to be a need to identify the underlying mechanisms that promote positive impacts within these interventions. This presentation describes the process of developing a hybrid realist and participatory evaluation to examine peer support services for youth with complex mental health and substance use challenges. The study took place in the context of the Transitional Aged Youth program implemented by LOFT Community Services that supports youth between the ages of 14-26. This program engages and utilizes young people who were previous clients of LOFT as peer support workers to help design and facilitate programming to support clients in developing life skills, autonomy and wellness, regardless of the complexity of the challenges they may be facing.

The focus of the presentation is threefold. First, we will discuss the development and dynamics of forming an engaging and ethical research partnership between primary investigator and peer researcher. Then we will discuss how this influenced the overall design of the study and related findings. Finally, we will review the lessons learned and implications for the findings and other related research. 